Pour les laits écrémés et pour les crèmes, nous recourons à un procédé spécial.

Pour la réaction mentionnée au commencement de ce travail, nous employons aussi de la poudre de charbon animal en suspension à 0,5-1\% dans de l'eau physiologique; les résultats obtenus sont bons, mais pas si bons qu'avec les globules rouges. Les quantités de lait et de suspension sont toujours les mêmes : $1 \mathrm{~cm}^{3}$ de lait et 1 goutte de la suspension de charbon.

Kонn et KLemm, de Carlsbad, ont confirmé nos recherches et ont donné à notre réaction le nom : La réaction de l'Anneau de SchernGorli; ces deux auteurs ont fait les mêmes expériences avec du carmin et de l'indigo et ils ont eu d'aussi bons résultats.

\title{
BIBLIOGRAPHIE
}

Schern et Gordr. - Archivos de la Sociedad de Biologia de Montevideo. Vol. V, $\mathrm{n}^{\circ} 3$, p. $246-49,1930$.

SCHERN et GoRLt. - Berliner Tierärztiche Wochenschrift. 1930, no 47, p. 893.

Конм et Кцемм. - Zeitschrift für Hygiene und Infections-Krankheiten der Haustiere. Tome 39, cahier $1 / 2$.

\section{SUR LE DIAGNOSTIC DU LAIT PASTEURISÉ A BASSE TEMPÉRATURE PAR L'ANNEAU DE SCHERN-GORLI}

\author{
par MM. le Dr F. G. KOHN et Ewald KLEMM, \\ de Carlsbad, République tchécoslovaque.
}

Il n'est pas difficile de reconnaître si un lait a été pasteurisé à des températures élevées, car nous avons à notre disposition des méthodes chimiques et biochimiques simples qui permettent de nous fixer sur ce point.

Au contraire, le diagnostic du lait pasteurisé à basse température présente toujours des difficultés et ce sont ces dernières qui, dans une certaine mesure, ont permis la critique de la pasteurisation à basse température, puisqu'en dehors du dénombrement microbien, nous n'avions aucun procédé sensible et sûr qui pût nous garantir que le lait avait été réellement chauffé à $63^{\circ} \mathrm{C}$. pendant une demi-heure.

Or, le contrôle des laiteries modernes qui travaillent à $63^{\circ} \mathrm{C}$. est indispensable. C'est dire que tout procédé susceptible de nous affirmer qu'il y a eu chauffage à $63^{\circ}$ sera le bienvenu.

Pour ces raisons, nous estimons que la méthode de l'anneau de SCHern-Gorli est du plus grand intérêt. Cette méthode se ramène en somme à l'étude de la direction des mouvements d'une suspension de globules rouges de cobaye dans le lait. Les résultats de leurs expé- 
riences ont montré qu'il y avait des différences remarquables entre le lait cru, le lait pasteurisé à basse température et le lait bouilli.

La méthode telle qu'elle a été décrite par ses créateurs n'est vraiment applicable que pour les laboratoires de sérologie, parce qu'elle exige une technique assez délicate et un nombre suffisant d'animaux d'expériences. Toutefois, dans leur première communication, ScHerN et GoRLI avaient déjà pensé à simplifier leur méthode, ce qu'ils ont fait en reconnaissant que l'on ponvait utiliser aussi bien du noir animal au lieu de globules rouges; nous avons été guidés par ces suggestions et voici dans quelle direction nous nous sommes engagés.

Nous avons l'intention de faire connaître ce que deviennent les mouvements des poudres insolubles colorées mises en suspension dans le lait ordinaire.

Nous avons employé de la poudre de noir animal, de carmin ou d'indigo, en suspension à $0,5-1 \%$ dans de l'eau ordinaire ou dans de l'eau physiologique. Nous avons ajouté 5 gouttes de ces suspensions à $5 \mathrm{~cm}^{3}$ de lait contenus dans des tubes de $1 \mathrm{~cm}$. de diamètre et de $8 \mathrm{~cm}$. de hauteur, à base plane, puis les tubes sont chauffés entre 30 et $36^{\circ} \mathrm{C}$. Si on ne les porte pas à cette température, la réaction se fait néanmoins, mais elle exige plus de temps.

Assez régulièrement, une partie de la poudre va au fond, ce qui est une circonstance sans valeur pour le jugement de la réaction. Nous attachons la plus haute importance à ce qu'une partie de la poudre s'élève dans le lait cru jusqu'au niveau de la crème pour former là un disque bien net se présentant comme un anneau coloré, que nous désignons comme anneau de Schern-Gorl. Le plus souvent, cet anneau se forme dans la première heure, au plus tard dans la seconde; au delà, les résultats sont plus douteux.

La congélation du lait, l'addition au lait, soit d'eau, soit de substances qui y sont introduites pour le conserver, ne gênent en rien la formation de l'anneau. Une communication personnelle du $\mathrm{D}^{\mathrm{r}}$ NESENI nous confirme ce qui vient d'être dit.

Lorsqu'on mélange du lait eru à du lait pasteurisé, l'anneau s'observe même lorsqu'il n'y a que $25 \%$ de lait cru ; mais ses dimensions sont moindres.

Quand le degré de l'acidification est élevé, la réaction est retardée ; mais avec le lait caillé ou près de se cailler, ainsi qu'avec la crème, il ne se fait aucun anneau.

Il est remarquable qu'avec le lait de chèvre, même cru, nous n'avons pas la réaction de l'anneau.

La température de $58 / 60^{\circ} \mathrm{C}$. fait disparaître le pouvoir du lait de former l'anneau, ainsi qu'il résulte de nos propres expériences et d'une communication personnelle du Dr NESENI. A fortiori, le lait pasteurisé à haute température et le lait bouilli ne donnent-ils pas la réaction. 
Un écrémage faible retarde la formation de l'anneau et la quantité de la poudre qui monte est diminuée. Quand l'écrémage est complet, l'anneau ne se forme plus. L'écrémage transforme le lait qui réagit, puisqu'il donne l'anneau, en un lait qui ne réagit plus et qui paraît, de ce fait, passer pour un lait qui pourrait être chauffé. Pour cette raison, nous pensons que les globules gras sont indispensables à la formation de l'anneau.

Avec le lait cru, quand les globules gras montent pour former la couche de crème, leur surface se charge de particules de poudre qui, en se rassemblant au niveau de la crème, va constituer l'anneau.

Le chauffage même à $58 / 60^{\circ} \mathrm{C}$., en amenant une altération de la surface des globules, fait perdre à ceux-ci leur pouvoir d'attraction pour les poudres qui ne sont plus entraînées vers le haut et se rassemblent en bas.

La réaction dite de l'anneau de Schern-Gorli donne des résultats assez marqués pour la pratique courante ; elle garantit la distinetion entre le lait cru et le lait pasteurisé à basse température. Un lait qui ne présente aucun anneau a donc été chauffé au-dessus de $58^{\circ} \mathrm{C}$. Il peut avoir été pasteurisé à basse tempéráture, à des températures élevées ou bouilli. Si nous obtenons un résultat négatif dans la recherche d'une pasteurisation à une température élevée chez un tel lait, e'est qu'il a été pasteurisé à basse température.

Les résultats de nos expériences sont publiés en détail dans Zeitschrift für Infectionskrankheiten, parasitäre Krankheiten und Hygiene der Haustiere, 1931, no 171-22.

SCHERN et GORLI. - Berliner tierzärztliche Wochenschrift, 1930, $\mathrm{n}^{\circ} 30$.

GorLI. - In Capitulos seleccionados sobre hï̆iene moderna de la leche, par le Prof.

Dr Kurt Schern, 2 Ed., Montevideo, 1931.

\section{VARIATIONS DE LA MATIËRE GRASSE DU LAIT DE VACHE DANS LA RÉGION D'ALGER}

(Contribution à l'étude de l'écrémage du lait) par MM.

L. MUSSO, et

Docteur ès sciences,

Professeur de Pharmacie à la Faculté mixte de Médecine et de Pharmacie d'Alger, Expert près la Cour et les Tribunaux d'Alger.

$$
\text { (Fin) }
$$

\section{INTERPRÉTATIONS DES RÉSULTATS ANALYTIQUES}

Le diagnostic de l'écrémage du lait est fait le plus souvent en prenant comme terme de comparaison une teneur moyenne considérée comme fixe. 